\title{
Preparation of a Novel Chitosan/Glycerol/Matrine Drug-Loading Film and the Study of Its Release Behavior in Vitro
}

\author{
Chunlei Zhang ${ }^{1,2, \dot{\dagger}, *}$, Jinghua $\mathrm{Li}^{3, \dagger}$ \\ ${ }^{1}$ Department of Stomatology, The First Affiliated Hospital, Jinan University, Guangzhou, China \\ ${ }^{2}$ School of Stomatology, Jinan University, Guangzhou, China \\ ${ }^{3}$ The Third Affiliated School of Clinical Medicine, Guangzhou University of Chinese Medicine, Guangzhou, China \\ Email address: \\ chunlei_zhang@yeah.net(ChunleiZhang) \\ ${ }^{*}$ Corresponding author \\ ${ }^{\dagger}$ Chunlei Zhang and Jinghua Li are co-first authors.
}

\section{To cite this article:}

Chunlei Zhang, Jinghua Li. Preparation of a Novel Chitosan/Glycerol/Matrine Drug-Loading Film and the Study of Its Release Behavior in Vitro. International Journal of Biomedical Materials Research. Vol. 6, No. 1, 2018, pp. 20-25. doi: 10.11648/j.ijbmr.20180601.14

Received: March 20, 2018; Accepted: April 2, 2018; Published: May 8, 2018

\begin{abstract}
Based on matrine (Mat), chitosan (CS) and carboxymethyl chitosan (CMC) as a plant source drug and a carrier material. A novel Mat/CS drug-loading film and Mat/CS/CMC drug-loading film were prepared. The mechanical properties, surface morphology and drug-loading capacity of the drug-loading film were characterized in vitro. The results showed that the mechanical strength had maximum value and the tensile modulus was up to $0.79 \mathrm{MPa}$ when the molecular weight of CS was 650,000 and the mass ratio of CS/glycerol was 1:1.4. The aggregate distribution of the Mat was located in the bottom surface of the film and was asymmetrical by SEM observation. Via release behavior tests in vitro, the drug-loading film showed high drug-loading capacity and long slowing-release properties. The release time of the drug-loading film increased along with the increase of CS molecular weight. When the bottom of the drug-loading film was coated with a layer of $1 \%$ carboxymethyl chitosan, the release times of the drug-loading film was increased to $108 \mathrm{~h}$. Thus, we conclude that the matrix materials of the drug-loading film are natural, green, nontoxic and biodegradable, and the preparation method is simple and avoid using a large number of organic solvents. The novel drug-loading film can obviously prolong release of drugs.
\end{abstract}

Keywords: Chitosan, Matrine, Drug-Loading Film, Release Behavior

\section{Introduction}

Oral mucosal ulcer is one of commonly seen oral diseases in clinic [1]. At present, the topical pharmaceutical dosage forms for treating oral mucosal ulcers mainly include liquid preparations, semi-solid preparations and solid preparations. The outstanding feature of these forms is fast-acting efficacy. However, as the duration of stay in mouth is short, the persistence is poor. In addition, as affected by saliva, local effective drug concentration is low, a good therapeutic effect cannot be achieved. Film is a new film-like formulation [2] made by dissolving drugs and evenly dispersed in the film-forming material developed in recent years. It can be applied to the skin or mucosal wound surface or ulcer surface, in this way the drug contained in the film can be absorbed directly through the mucosa, so the absorption of drugs is improved to achieve good therapeutic effect.

Because of its nontoxic and biodegradable, good drug compatibility and film-forming[3], good anti-inflammatory hemostasis [4] and antibacterial sterilization [5], tissue healing promoting and other pharmacological effects $[6,7]$, chitosan (CS) is widely used in the medical field. Chitosan sustained-release film is a film of a several millimeters made through evenly dispersing drugs in chitosan solution. It is mainly used in skin and mucosal wound or ulcer surface to promote wound healing, which increased the film-related performance and improved drug release. However, the short duration of effect of chitosan sustained-release film limits its application in the field of medicine. 
Matrine (Mat) is tetracyclic quinolizidine drugs [8, 9], widely presented in leguminous plants as Sophora flavescens, Sophora alopecuroides and Sophora subprostrata. Mat has a wide range of pharmacological effects such as anti-inflammatory, anti-hepatic fibrosis, analgesia and antiarrhythmic and is widely used in clinic [10-12].

Targeting as preparation of oral drug-loading film to extend the drug release time, selecting chitosan as film carrier, adding glycerol and matrine to prepare oral drug-loading film, this study investigated the mechanical properties, swelling degree, drug microscopic morphology and in vitro drug release rate by coating the bottom of the drug-loading film with carboxymethyl chitosan (CMC) solution to control the release rate of Mat.

\section{Materials and Methods}

\subsection{Main Reagents and Instruments}

Reagents: Chitosan (CS), degree of deacetylation $>85 \%$, Jinan Haidibei Pharmacy Technology Co., Ltd.; Mat, 98\%, Xi'an Zhongxin Pharmaceutical Technology Co., Ltd.; (CMC), the average relative molecular mass of $6.1 \times 10^{4}$, the degree of carboxymethyl substitution of $66 \%$, Jinan Haidibei Ocean Pharmaceutical Engineering Co., Ltd.; acetic acid, analytical grade, Guangzhou Chemical Reagent Factory; glycerol, analytical grade, Guangzhou Chemical Reagent Factory.

\subsection{Degradation of Chitosan}

Chitosan dissolved in $1 \%$ acetic acid solution to $2 \% \mathrm{CS}$ solution, then ozone was added for degradation experiments. Viscosity was used to investigate the viscosity-average relative molecular mass of $\mathrm{CS}$ at different degradation times $(15,30,45,60,75,90,120 \mathrm{~min})$. CS relative molecular mass were investigated in different degradation times as: 650,000, $580,000,480,000,310,000,220,000,100,000,75,000$ and 56,000 respectively.

\subsection{Preparation of CS Film, Mat/CS Film and Mat/CS/CMC Film}

Five sets of $10 \mathrm{~g}$ of the aforesaid CS solution with different relative molecular masses were respectively prepared, each adding $0.2,0.24,0.28,0.32$ and $0.36 \mathrm{~g}$ glycerol to make solution with $\mathrm{CS} /$ glycerol mass ratio of 1: 1, 1: 1.2, 1: 1.4, 1: 1.6, 1: 1.8. After being completely dissolved, CS films with different relative molecular mass and glycerol content were formed with an amount of 40 pieces by drying at room temperature.

CS with relative molecular mass of $310,000,480,000$ and 650,000 , and CS/glycerol mass ratio of 1:1.4 were selected, each adding proper glycerol and Mat to prepare Mat/CS solution with Mat content of $6 \%, 8 \%, 10 \%, 12 \%$ and $14 \%$ respectively. After being completely dissolved, Mat/CS films with different relative molecular mass and different Mat content were formed with an amount of 15 pieces by drying at room temperature.

Three films of CS with relative molecular mass of 650,000 ,
CS/glycerol mass ratio of 1: 1.4 and Mat content of $12 \%$ were selected. CMC solutions with concentrations of $0.5 \%$ and $1 \%$ respectively were coated at the bottom of the films and were marked 1 \# film and 2 \# film respectively; CMC solutions with $0.5 \%$ concentrations were coated at the front and bottom of the film was marked $3 \#$ film. After being dried at room temperature, Mat / CS / CMC drug-loading films were formed.

\subsection{Physical Properties of CS Film}

CS Film macroscopic morphology observation: After pouring into film, the surface morphology of the film is directly observed with naked eyes. Mechanical properties test of CS film: films were prepared with CS being degraded for 0 , $30,45 \mathrm{~min}$. Testing is done at a tensile rate of $1 \mathrm{~mm} / \mathrm{min}$. Each sample was tested three times repeatedly to calculate modulus and elongation at break. Swelling degree test of CS film: 0, 30, $45 \mathrm{~min}$ of CS prepared into films, weight of which were recorded as $\mathrm{m} 0$; after films were soaked in saline for $3 \mathrm{~d}$, the surface moisture was blotted up with filter paper, the relative proton mass of which were recorded as $\mathrm{mL}$.

\subsection{Macroscopic and Microscopic Morphology of Mat/CS Film}

Macroscopic morphology observation of Mat/CS film: After pouring into film, the surface morphology of the film is directly observed with naked eyes. Microscopic morphology observation of Mat/CS film: CS with relative molecular mass of 480,000 and 650,000 respectively and Mat with mass percentage content of $10 \%$ and $12 \%$ respectively were selected to prepare Mat/CS drug-loading film samples. After spraying gold, the front and bottom surface morphology were observed by SEM.

\subsection{In Vitro Release Behavior of Mat/CS Film and Mat/CS/CMC Film Test}

CS with relative molecular masses of $310,000,480,000$ and 650,000 were respectively prepared as CS blank films and drug-loading films with Mat content of $0 \%$ and $12 \%$ (marking $1 \#$ film, $2 \#$ film and $3 \#$ film respectively). $100 \mu \mathrm{L}$ solution each were taken out after soaking $0.5,1,2,3,5,9,13,23,37$, $61,85,109,133,157 \mathrm{~h}$ and were placed in a separatory funnels, adding $10.00 \mathrm{~mL}, 2 \times 10-4 \mathrm{~mol} / \mathrm{L}$ bromothymol blue buffer ( $\mathrm{pH}$ 7.6) and $10.00 \mathrm{~mL}$ chloroform successively, shaking up, standing about $2 \mathrm{~h}$ until water and chloroform were complete delaminated, filtering off chloroform. The absorbance of the solution at $415 \mathrm{~nm}$ were measured. The measured absorbance was substituted into the standard curve to calculate Mat drug concentration at different times.

\section{Results}

\subsection{Effect of Chitosan Relative Molecular Mass on Mechanical Properties of CS Film}

With the decrease of chitosan relative molecular mass, the 
modulus of CS film decreased and the elongation at break increased successively. When the relative molecular mass of chitosan was 650,000 , the modulus of CS film was the largest $(0.7875 \mathrm{MPa})$, while the elongation at break was the smallest $(17.3 \%)$, the stress-strain curve showed obvious hard and strong characteristics. When the relative molecular mass of chitosan was 310,000 , the modulus of CS film was the smallest $(0.1471 \mathrm{MPa})$, while the elongation at break was the largest $(43.3 \%)$, and the stress-strain curve showed soft and tough characteristics.

\subsection{Swelling Degree of CS Film and Mat/CS/CMC Film}

The drug release rate of chitosan-loading film is closely related to the swelling degree. Therefore, the swelling degree in physiological saline of CS film, prepared by different relative molecular mass $\mathrm{CS}$, and Mat/CS/CMC drug-loading films were investigated. The swelling degree of CS film prepared by CS with molecular weights of $650,000,480,000$, 310,000 were $0.0743,0.2133$ and 0.3051 respectively. It can be seen that the swelling degree of CS film decreases with the increase of CS molecular weight; the swelling degree of \# 1 film, \# 2 film and \# 3 film of Mat/CS/CMC drug-loading films were $0.8142,0.9314$ and 1.1654 respectively. With the increase of the $\mathrm{CMC}$ concentration, the swelling degree of the drug-loading film increased, and the swelling degree of the drug-loading film with CMC-coated on both the top and bottom surfaces was significantly higher than that of the single side-coated drug-loading film.

\subsection{Macroscopic Morphology of Mat/CS}

The appearance of Mat/CS film with CS relative molecular mass of 650,000 is whiter, but the Mat/CS film with relative molecular mass of 310,000 is darker in color. It indicates that ozone degradation will darken the color of chitosan solution.

The appearance of CS films with different Mat content prepared by CS of different relative molecular mass $(310,000$, 480,000 and 650,000) showed that Mat/CS films with relative molecular weight of 650,000 are all white while the Mat/CS films with relative molecular mass of 480,000 and 310,000 are light yellow. When the Mat content was over 14\%, the Mat/CS film tended to precipitate oil after drying. Therefore, it can be judged that the maximum content of Mat drug in the film was $12 \%$.

\subsection{In vitro Release Behavior of Mat/CS Film and Mat/CS/CMC Drug-Loading Film}

By UV spectrophotometer, we found that the Mat standard working curve, fitting curve is $\mathrm{C}=-23.056+42.901 \mathrm{~A}, \mathrm{r}=0$. 9995. According to the standard curve, we studied the in vitro release behavior of Mat/CS film prepared by CS with relative molecular mass of 310,000, 480,000 and 650,000 (Mat mass content was $12 \%$ ) respectively. The results are shown in Figure 1.

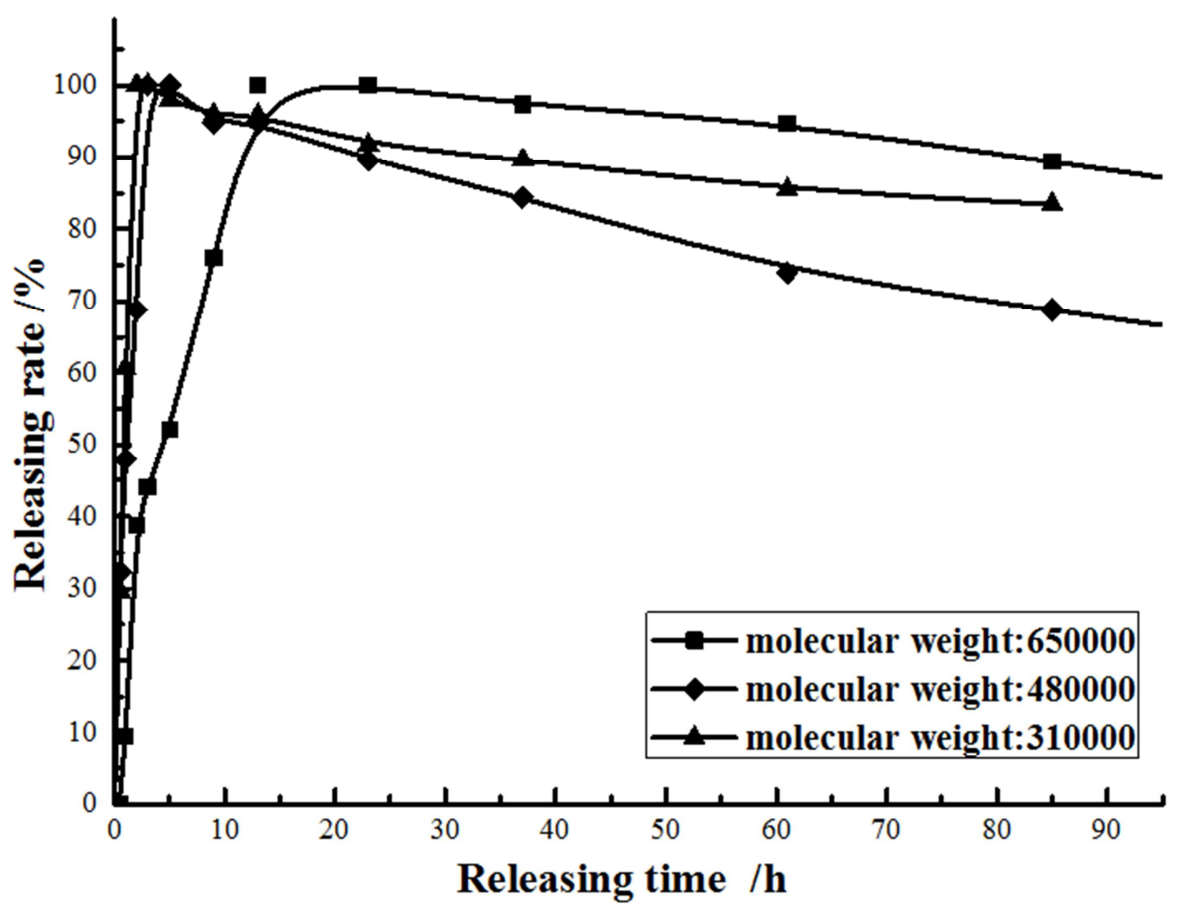

Figure 1. Drug release behavior in vitro of Mat/CS films (Mat content: 12\%).

As can be seen from Figure 1, the drug of Mat/CS film with relative molecular mass of 650,000 was completely released at $23 \mathrm{~h}$, that of Mat/CS film with relative molecular mass of 480,000 was completely released at $13 \mathrm{~h}$ and that of Mat/CS film with relative molecular mass of 310,000 was completely released at $9 \mathrm{~h}$. This showed that the relative molecular mass of chitosan had a significant effect on the in vitro release of Mat / CS film. The smaller the relative molecular mass of chitosan was, the faster the release of drug in drug-loading film.

According to in vitro release behavior (Figure 2) of $\mathrm{Mat} / \mathrm{CS} / \mathrm{CMC}$, the drug release rate can significantly be 
delayed after coating CMC solution on the Mat/CS film. The Mat/CS film without CMC solution was completely released in $23 \mathrm{~h}$, while the 1 \# film coated with $0.5 \%$ CMC solution on the bottom was completely released in $60 \mathrm{~h}$. The effect of delay release was very obvious. In addition, the \# 2 film coated with $1 \% \mathrm{CMC}$ solution on the bottom was completely released in $108 \mathrm{~h}$. It can be inferred that the concentration of $\mathrm{CMC}$ solution has a great impact on drug release rate, the larger the $\mathrm{CMC}$ concentration is, the slower the drug releases.

If a layer of concentration $0.5 \% \mathrm{CMC}$ solution was coated on both the front and bottom of $3 \#$ film, the drug was completely released in $60 \mathrm{~h}$, almost same as $1 \#$ film. This indicated the delay effects on drug release were basically the same to coat CMC solution on both sides of the film and on only the bottom of film. This may be related to the phenomenon of drug deposition on the bottom observed by SEM. Because the drug is deposited on the bottom of the drug-loading film during the film-forming process, coating CMC solution on the bottom can effectively delay the release of the drug, while coating CMC solution on the front cannot release substantially.

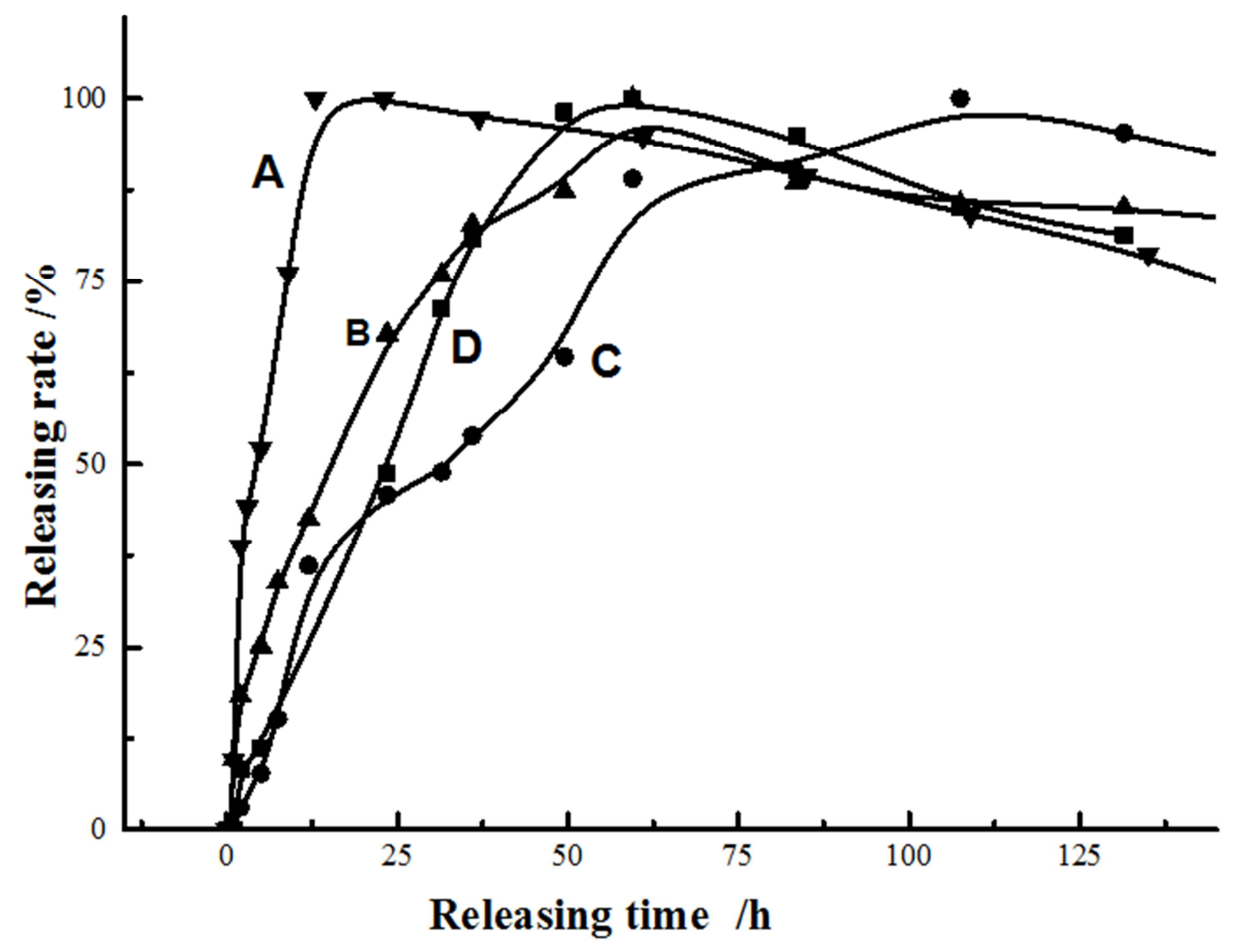

Figure 2. Release behavior in vitro of Mat/CS/CMC films. A: Blank film; B: No.1 film; C: No.2 film; D: No.3 film.

\subsection{Microscopic Morphology of Drug-loading Film}

The microscopic morphology of Mat/CS film was observed by SEM to investigate the drug dispersion in CS film. Figures 3 5 show the SEM of Mat/CS films prepared with different CS relative molecular mass and different Mat contents respectively.

As can be seen from Figure 3, no drug particle appeared on the air-facing surface of CS film, while more drug particles appeared on the side facing the substrate, indicating that Mat gradually crystallized out of the water as the water evaporated. By virtue of gravity, the drug will deposit towards the bottom of film, more crystalline particles will appear at the bottom. In addition, the sizes of Mat crystalline particles are within the micrometer, submicron or nanometer range.

Drug-loading films with different Mat content prepared by CS of different relative molecular masses all showed a phenomenon of drug aggregating on the film bottom (Figure 4 and 5). This shows that the drug dispersion in Mat/CS films is asymmetric. In fact, this asymmetric distribution is beneficial to the medical use. For example, in the course of using as an anti-inflammatory excipient, drug-loading film with more drug facing the affected area may be more concentrated to exert better curative effect.

\section{Discussion}

Using natural degradable CS and its derivatives as matrix materials, matrine as plant-derived drug and drug substance glycerol as plasticizer, Mat/CS and Mat/CS/CMC drug-loading films were the first time systematically prepared through solution casting method. The effects of CS molecular weight, mechanical properties, swelling degree and microscopic morphology on the properties of drug-loading films were investigated. (1) The optimum preparation conditions were obtained by orthogonal experiment: drug-loading films, prepared when the mass ratio of CS to glycerol was 1: 1.4, the CS molecular weight was from 310000 to 650000 and the matrine content was 5\%, own good physical properties. (2) SEM observed that Mat drug accumulated on the bottom of the drug-loading film and presented an asymmetric distribution. (3) The matrix materials used to prepare drug-loading film are natural, green, nontoxic 
and biodegradable, and the preparation method is simple and easy without using large quantities of organic solvents. Hence, the film is expected to be used as a carrier drug-loading film material for oral ulcers.

The in vitro release behaviors of Mat/CS drug-loading film and Mat/CS/CMC drug-loading film loaded with plant-derived drugs were further studied. The results showed that: (1) CS molecular weight affected the release of drug-loading film. The smaller the molecular weight was, the faster the drug released, release time can be reaching $23 \mathrm{~h}$. (2) Applying CMC on the surface of Mat/CS drug-loading film can delay the release of drug. For example, applying a layer of $1 \% \mathrm{CMC}$ solution on the bottom can delay the release time from $23 \mathrm{~h}$ to $108 \mathrm{~h}$. The sustained release efficacies of the prepared Mat/CS drug-loading films and Mat/CS/CMC drug-loading films were significantly higher than those reported in some of the reported chitosan derivative drug-loading films[13].
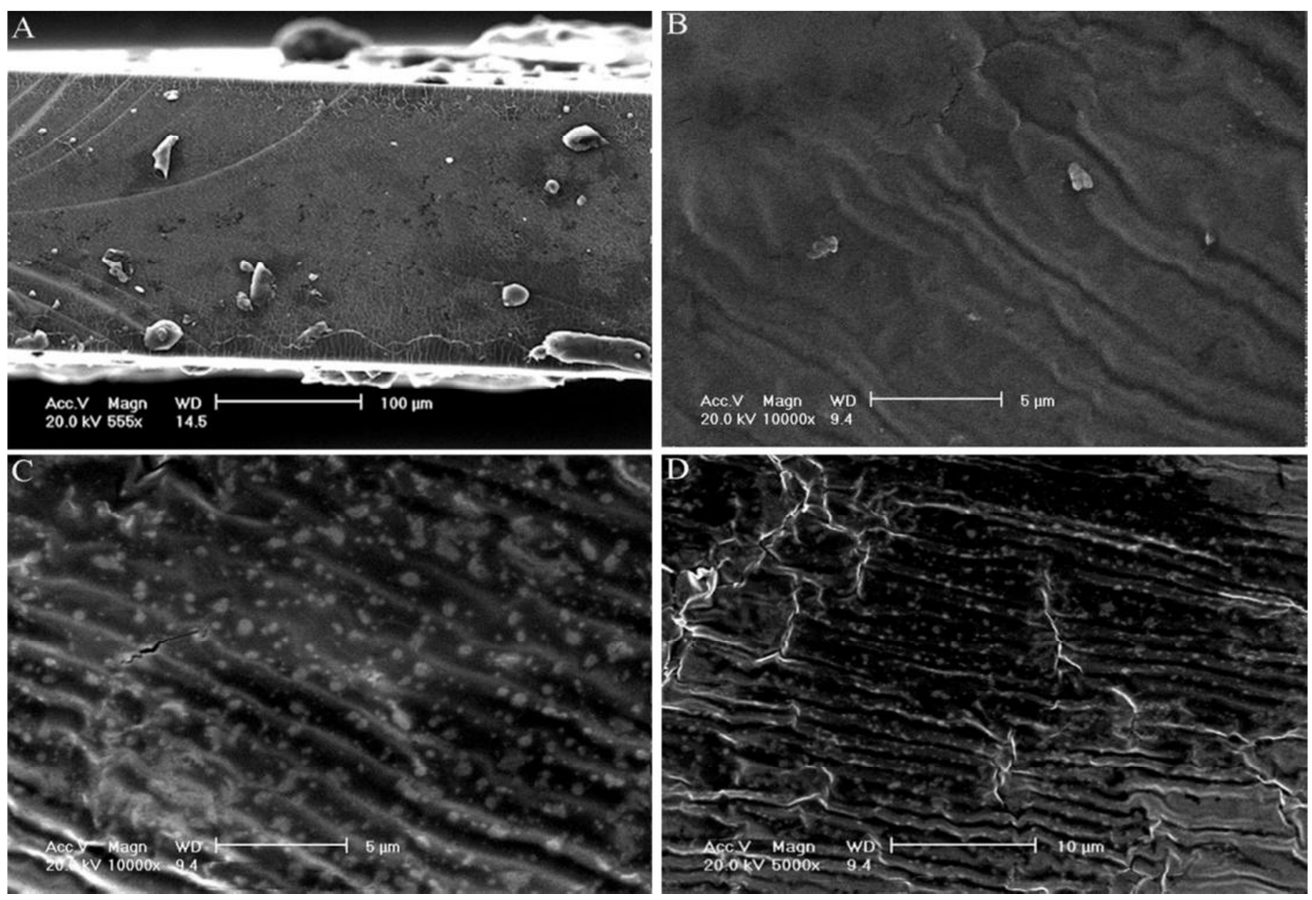

Figure 3. SEM of Mat/CS films (CS molecular weight: 650000, Mat content: 12\%). A: Fracture surface; B: Front surface; C and D: Undersurface.
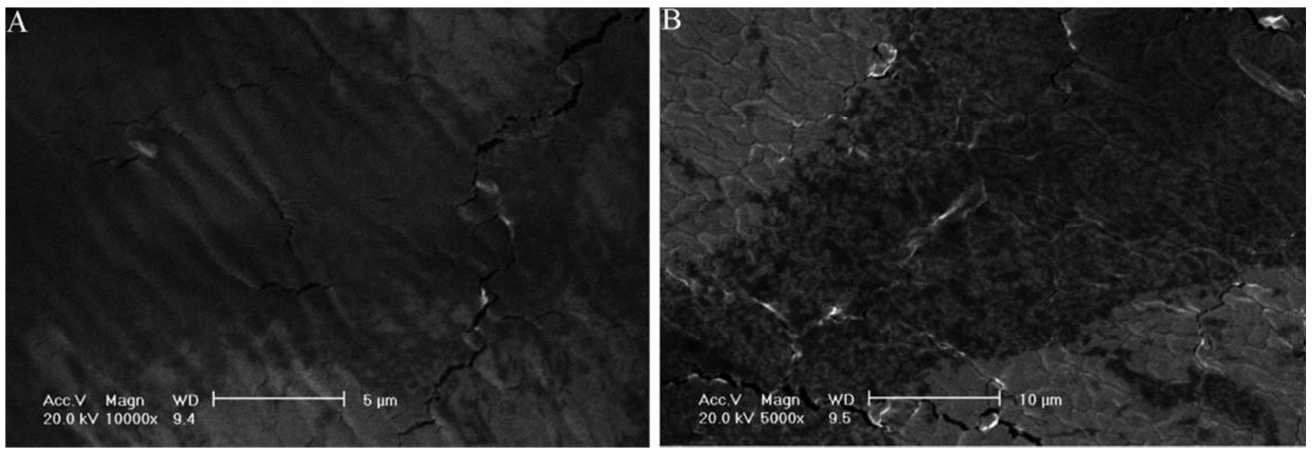

Figure 4. SEM of Mat/CS films (CS molecular weight: 650000, Mat content: 10\%). A: Front surface; B: Undersurface.
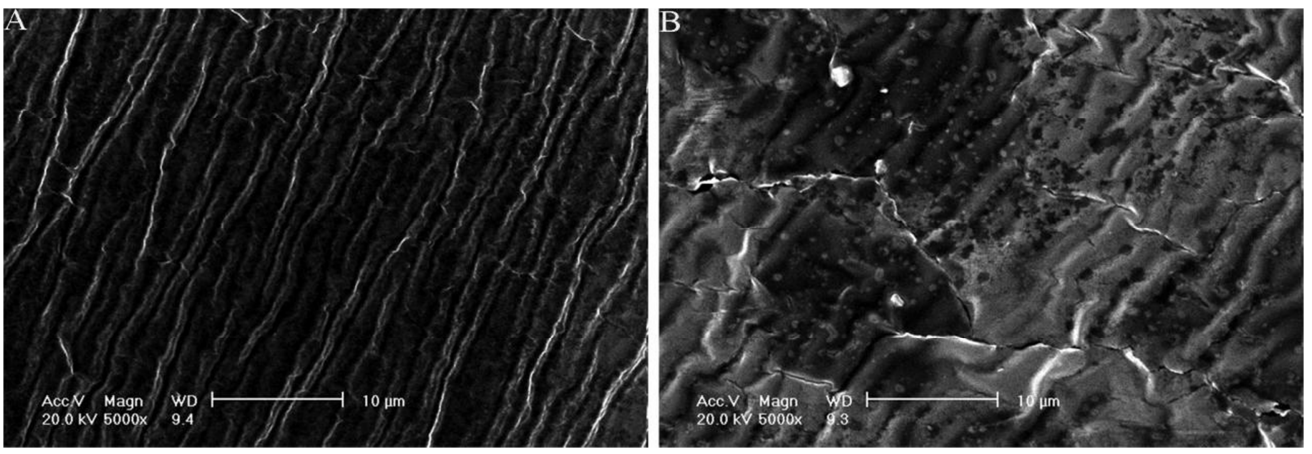

Figure 5. SEM of Mat/CS films (CS molecular weight: 480000, Mat content: 12\%). A: Front surface; B: Undersurface. 


\section{Acknowledgements}

The authors declare no conflict of interest.

\section{References}

[1] S. Hamedi, O. Sadeghpour, M. R. Shamsardekani, G. Amin, D. Hajighasemali, Z. Feyzabadi, The Most Common Herbs to Cure the Most Common Oral Disease: Stomatitis Recurrent Aphthous Ulcer (RAU), Iran Red Crescent Med J, 2016, (18): e21694.

[2] P. Yue, M. Xiao, Y. Xie, Y. Ma, Y. Guan, Z. Wu, P. Hu, Y. Wang, The Roles of Vitrification of Stabilizers/Matrix Formers for the Redispersibility of Drug Nanocrystals After Solidification: a Case Study, AAPS PharmSciTech, 2016, (17): 1274-1284.

[3] G. F. Caetano, M. A. Frade, T. A. Andrade, M. N. Leite, C. Z. Bueno, A. M. Moraes, J. T. Ribeiro-Paes, Chitosan-alginate membranes accelerate wound healing, J Biomed Mater Res B Appl Biomater, 2015, (103): 1013-1022.

[4] M. B. Dowling, R. Kumar, M. A. Keibler, J. R. Hess, G. V. Bochicchio, S. R. Raghavan, A self-assembling hydrophobically modified chitosan capable of reversible hemostatic action, Biomaterials, 2011, (32): 3351-3357.

[5] J. F. Barata, R. J. Pinto, V. I. Vaz Serra, A. J. Silvestre, T. Trindade, M. G. Neves, J. A. Cavaleiro, S. Daina, P. Sadocco, C. S. Freire, Fluorescent Bioactive Corrole Grafted-Chitosan Films, Biomacromolecules, 2016, (17): 1395-1403.

[6] J. X. Chen, H. H. Shen, M. Niu, Y. M. Guo, X. Q. Liu, Y. Z.
Han, Y. M. Zhang, Y. L. Zhao, B. K. Bai, W. J. Zhou, X. H. Xiao, Anti-hepatitis B virus effect of matrine-type alkaloid and involvement of p38 mitogen-activated protein kinase and tumor necrosis factor receptor-associated factor 6, Virus Res, 2016, (215): 104-113.

[7] G. L. Zhang, L. Jiang, Q. Yan, R. H. Liu, L. Zhang, Anti-tumor effect of matrine combined with cisplatin on rat models of cervical cancer, Asian Pac J Trop Med, 2015, (8): 1055-1059.

[8] J. Huang, H. Xu, Matrine: Bioactivities and Structural Modifications, Curr Top Med Chem, 2016, (16): 3365-3378.

[9] J. Yong, X. Wu, C. Lu, Anticancer Advances of Matrine and Its Derivatives, Curr Pharm Des, 2015, (21): 3673-3680.

[10] W. C. Lin, C. C. Lien, H. J. Yeh, C. M. Yu, S. H. Hsu, Bacterial cellulose and bacterial cellulose-chitosan membranes for wound dressing applications, Carbohydr Polym, 2013, (94): 603-611.

[11] M. J. Simoes, A. Gartner, Y. Shirosaki, R. M. Gil da Costa, P. P. Cortez, F. Gartner, J. D. Santos, M. A. Lopes, S. Geuna, A. S. Varejao, A. C. Mauricio, In vitro and in vivo chitosan membranes testing for peripheral nerve reconstruction, Acta Med Port, 2011, (24): 43-52.

[12] S. Simsek, H. I. Canter, E. Konas, P. Korkusuz, D. Demir, F. Oner, I. Unsal, M. E. Mavili, A new concept in treatment of burn injury: controlled slow-release granulocyte-monocyte colony-stimulating factor chitosan gel system, Ann Plast Surg, 2011, (67): 583-588.

[13] L. L. Zhang, P. Li, Y. M. Li, A. Q. Wang, Preparation and characterization of magnetic alginate-chitosan hydrogel beads loaded matrine, Drug Dev Ind Pharm, 2012, (38): 872-882. 